\title{
Renal and Visceral Sutureless Anastomosis During Thoracoabdominal Aortic Repair
}

\author{
Andrea Kahlberg, Matteo Bossi, Daniele Mascia, \\ Enrico Rinaldi, Yamume Tshomba, \\ Germano Melissano, and Roberto Chiesa
}

\subsection{Introduction}

In spite of multiple technical improvements in the surgical treatment of thoracoabdominal aortic aneurysm (TAAA) that lead to significant morbidity and mortality reduction, visceral and renal ischemic complications are still frequent, even in the most specialized centers [1].

As a matter of fact, perioperative acute kidney dysfunction still complicates up to $70 \%$ of these procedures, and the consequences of postoperative renal failure are remarkable. They represent the strongest predictor of early mortality and are

Electronic Supplementary Material The online version of this chapter (doi:10.1007/978-3-319-94761-7_19) contains supplementary material, which is available to authorized users.

\footnotetext{
A. Kahlberg $(\bowtie) \cdot$ M. Bossi $\cdot$ D. Mascia $\cdot$ E. Rinaldi G. Melissano

Vascular Surgery, Vita-Salute University

School of Medicine, San Raffaele Scientific Institute, Milan, Italy

e-mail: kahlberg.andrea@hsr.it

Y. Tshomba

Unit of Vascular Surgery, Fondazione Policlinico Universitario A. Gemelli IRCCS, Università Cattolica del Sacro Cuore, Rome, Italy
}

Unit of Vascular Surgery, San Raffaele Scientific Institute, Vita-Salute University, Milan, Italy

R. Chiesa

Unit of Vascular Surgery, San Raffaele Scientific Institute, Vita-Salute University, Milan, Italy associated with permanent long-term chronic kidney disease $[2,3]$.

Multiple etiopathological factors are supposed to be involved in perioperative visceral and renal dysfunction: the most important, following many authors' opinions, are the time of organ ischemia during artery reattachment and the associated atherosclerotic vessel disease, such as ostial stenosis or dissection. These factors account for a significant increase in technical difficulty, prolonged time of anastomosis, and increased global risk of intraoperative organ injury.

In the past, the first "sutureless anastomoses" performed on visceral aortic branches (including visceral and renal arteries) were performed during debranching procedures, using covered selfexpanding stents [4]. The goal was to obviate the need for technically demanding vessel exposure and challenging anastomoses, thus obtaining reduction of flow interruption and simplifying the performance of complex aortic repair.

The Gore Hybrid Vascular Graft (GHVG; WL Gore and Associates, Flagstaff, Ariz) represents the linear evolution of this technique: a novel expanded polytetrafluoroethylene (ePTFE) vascular prosthesis is associated with a nitinolreinforced covered self-expanding section at one of its extremities that allows to perform "sutureless" endovascular anastomosis at one side and a standard surgical suture on the other side of the prosthesis. 
In this chapter, we describe this novel device and our experience with this graft for renal and visceral revascularization during TAAA open repair, in order to assess the safety and short-term results of this technique as compared to standard revascularization strategies.

\subsection{General Considerations}

Open surgical repair for thoracoabdominal aortic disease, such as atherosclerotic aneurysms or dissection, is durable with well-defined and acceptable long-term survival [5]. With open surgical procedures, the widest range of aortic anatomies and diseases can be covered. In spite of specific renal and visceral protection strategies, acute kidney dysfunction still remains one of the most serious complications of TAAA open repair, with around $6-10 \%$ of patients requiring postoperative hemodialysis $[2,6]$. Considering lesser grades of postoperative kidney injury, an up to $50 \%$ elevation in baseline creatinine level may be observed in more than $70 \%$ of patients [1] who underwent open surgical repair of TAAA.

In this regard, an important consideration must be undertaken: in a recent study reviewing all the published data of the past decade (2000-2010) for open TAAA surgical repair [7], the rate of postoperative renal complications did not seem to decrease if compared with a similar review published in 1995, regardless of medical and surgical advances [8].

Major causes of intraoperative renal damage include temporary ischemia during aortic cross-clamping and visceral vessels repair, arterial embolism, and stenosis or dissection of the reattached renal vessel [9-11]. These harmful mechanisms are known to develop especially when the quality of the arterial wall is poor, with severe atherothrombotic vessel degeneration, and when the surgical reattachment of the renal artery is demanding because of its anatomical location.

Methods usually employed for renal artery revascularization during TAAA open repair include direct reattachment on the aortic graft using the "Carrel patch technique," described by Crawford et al. (usually together with other visceral vessels or even by direct single-vessel reimplantation) [12], or the use of a single aortorenal bypass.

The inclusion of many vessels in a visceral aortic patch (VAP) is relatively simple and carries two important advantages: decreasing the global number of anastomoses and, most of all, the duration of organ ischemia. However, a fragile aortic wall (such as in dissected patients or in Marfan's) can cause difficulties with bleeding control, the suture line may become too long in case of considerable distances between the visceral arterial ostia, and the aortic patch tissue may result in subsequent aneurysmal degeneration in time [13]. The latter is considered the main disadvantage of the VAP technique: we previously demonstrated that a large VAP including four visceral vessels is associated with VAP aneurysm formation [13] and that consequent corrective surgery is associated with high morbidity and mortality [14].

To obtain a reduction in VAP size, separate reattachment of distant visceral vessels must be considered, directly or with the interposition of a graft. The vessel is usually indicated for a separated reattachment in the left renal artery. A single aorto-renal/visceral graft interposition allows better bleeding control, may accommodate several anatomies, and presents reduced risk of late aneurysmal degeneration. Its major disadvantage is that two anastomoses are required for each vessel revascularization, often making this option time-consuming and occasionally challenging. Alternatively, the use of presewn aortic branched grafts carries the advantage of reducing the number of total anastomoses to be performed, consequently decreasing operative times [15]. The negative aspect of this graft is that branches are presewn to the aortic graft in a fixed position and may not adapt well to the patient's anatomy leading to subsequent kinking or twisting after viscera derotation.

Finally, customized branched aortic graft may be employed, in which prosthetic ramifications are sewn during the operation prior to aortic cross-clamping, considering the variable anatomy of each single patient [16]. Although this strategy may theoretically represent a valuable alternative in order to reduce the visceral ischemic time (but definitely increasing total operative time), it is quite difficult to predict a priori how the aortic 
graft will arrange after performing the proximal, distal, and intercostal patch anastomoses.

In relation to atherosclerotic disease, or caused by aortic dissection, patients with TAAAs often present an occlusive disease involving the visceral branches. The presence of calcification and thrombus at the origin of the renal or visceral arteries is associated with a high risk of plaque disruption and dissection during vessel reattachment, leading to visceral malperfusion [17], and still represents a significant predictor of renal or visceral complications after TAAA repair [18]. Despite the fact that artery endarterectomy may be required before revascularization to improve patency rates, it represents a high-risk maneuver with important limitations, including the risk of vessel thrombosis, distal dissection, and vessel perforation due to the friable endarterectomized wall [19].

To overcome these difficulties, several groups started using bare stents during TAAA open repair, positioned and expanded within the artery under direct vision, in order to both treat arterial stenosis or dissection avoiding endarterectomy and also to tack down an unsatisfactory endpoint after visceral endarterectomy [20-22].

The use of covered self-expanding stents for "sutureless anastomoses" in challenging anatomies has been reported by several authors. First among all, Lachat et al. reported the concept of a Viabahn-assisted (WL Gore and Associates, Flagstaff, Ariz) sutureless anastomosis in the so-called "VORTEC" technique. The author proposed this technique for visceral revascularization during debranching procedures in hybrid complex aortic repair [4]. The distal end of the covered stent was inserted over a guidewire into the visceral vessel, and the proximal end was sutured end to side directly with the main graft or was fixed into an interposition branch graft on the main bypass. In 2009, the same group published the results of 58 patients submitted to hybrid repair of TAAA or pararenal aortic aneurysm, using the VORTEC technique to revascularize 98 renal and 15 visceral arteries, reporting an overall primary patency rate of $97 \%$ at 22 months [23]. They also applied this technique to debranching procedures of supra-aortic trunks, reporting a midterm $91 \%$ patency rate without implantation-related complications [24]. Another setting in which this technique can be employed, as described by several groups, is for femoropopliteal revascularization $[25,26]$.

The GHVG represents the natural evolution of these procedures with a tubular heparin-bounded ePTFE graft pre-connected to the nitinol stent-reinforced section, guaranteeing in-line flow while avoiding flow turbulence. Excluding the literature regarding hemodialysis access, just three case reports have been published to date reporting the use of this novel graft: Levack et al. reported its use for rapid left common carotid revascularization during aortic arch debranching [27], Nigro et al. used the GHVG to perform a sutureless distal anastomosis during surgery for a high-lying internal carotid artery aneurysm [28], and Bornak et al. reported a modified use for celiac trunk and superior mesenteric artery revascularization during a visceral debranching procedure, with satisfying early patency [29].

In our experience of TAAA open repair with the use of GHVG for remote or hostile vessels, many advantages must be underlined:

1. The ability to perform the aorto-renal bypass in a faster and easier manner, reducing ischemic renal time during cold perfusion

2. Low-risk rate and less time to access to remote ostium locations, typically the right renal artery, avoiding extensive preparation and reducing connected complications

3. Possibility to obtain high conformability of the graft, resulting in less kinking or twisting of the revascularized vessels upon viscera derotation

4. Helpful in cases of stenotic or dissected vessels, avoiding the need for additional surgical (endarterectomy) or endovascular procedures (bare stenting)

5. Great applicability for all the visceral and renal vessels (also for the celiac trunk), as long as they respect minimum requirements in length and diameter

6. Possibility to obtain a customized reconstruction in complex anatomy

7. Direct flow into the vessel, with high reduction in turbulence of the blood flow 
Fig. 19.1 The Gore Hybrid Vascular Graft (GHVG). To notice: the ePTFE standard vascular prosthesis associated with a closed covered selfexpandable stent-graft and the linear delivery system

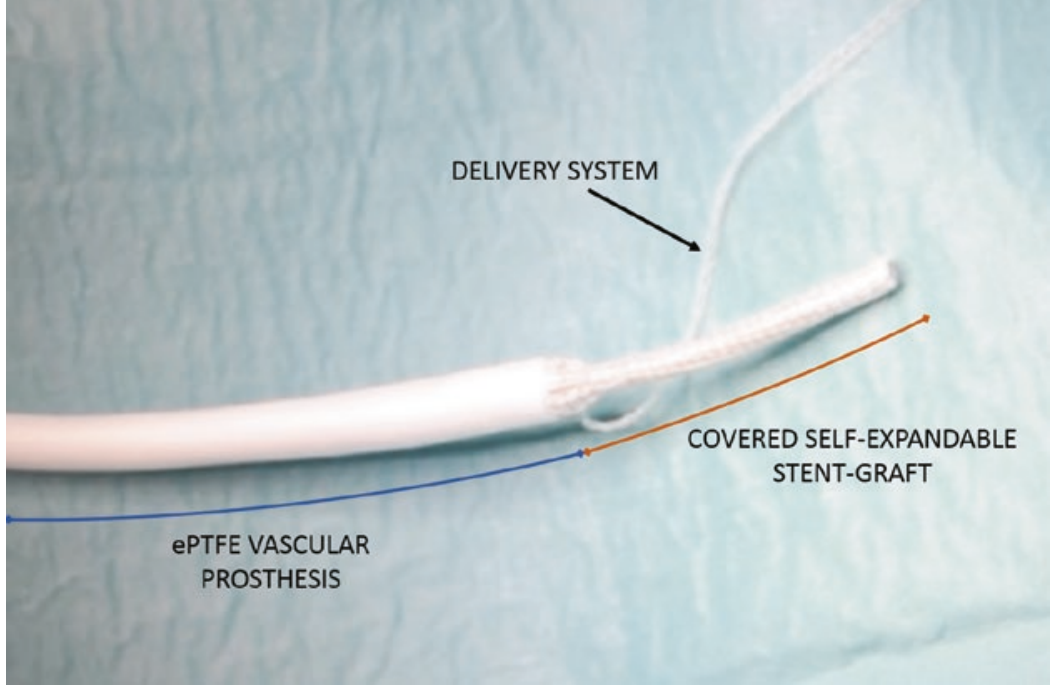

\subsection{Device Description}

The Gore Hybrid Vascular Graft (GHVG) is an expanded polytetrafluoroethylene (ePTFE) vascular prosthesis that has a distal section reinforced with nitinol. The nitinol-reinforced section is partially constrained to allow for easy insertion and deployment into a vessel. The graft has a continuous lumen bonded with the CARMEDA $^{\circledR}$ BioActive Surface, consisting of a stable covalently bonded reduced molecular weight heparin of porcine origin. An embedded low-permeability film provides a barrier to ultrafiltration.

This graft received the United States (US) Food and Drug Administration (FDA) approval on March 2010 and obtained the CE mark in July 2012. This device is currently commercially available in the USA, Europe, Russia, and few Middle Eastern countries. At the time of writing, this device is approved in the European market for hemodialysis access, lower-limb bypass grafting, aortic arch debranching, and visceral revascularization [30].

The prosthesis is available with the vascular graft section length of $50 \mathrm{~cm}$ and a fixed diameter of $6 \mathrm{~mm}$; the nitinol-reinforced section instead presents length of 5 and $10 \mathrm{~cm}$ with diameter of $6,7,8$, and $9 \mathrm{~mm}$.

The delivery system consists of a line that, once pulled, releases the constrained graft. Deployment of the nitinol-reinforced section will occur from the tip of the constraint graft toward the non-nitinol section. Correct deployment of the nitinol-reinforced section does not entail a shortening of the graft. Once deployment has started, repositioning of the nitinol section should not be attempted (Fig. 19.1).

\subsection{Indications and Contraindications}

In the initial part of our experience, indication for the use of the GHVG was generally limited to renal vessels. Once the real efficacy of these devices had been verified, their use has been expanded to other visceral vessels, both in open and hybrid TAAA surgical reconstructions.

Generally, our indications to the use of the GHVG are:

- Vessels which were not considered suitable for inclusion in an aortic patch, because of their anatomical location or the poor quality of the surrounding aortic wall

- Anatomical location of the artery ostium considered challenging for performing a conventional anastomosis with a standard single bypass

- Severe atherosclerotic disease of the proximal tract of the artery (including the presence of highly calcified or disrupted plaque, critical stenosis, or local dissection) 
The choice to use the GHVG was guided by the intention to decrease technical complexity and duration of the distal anastomosis, reduce accidental damage to the arterial wall, concurrently treat stenotic or dissected arteries, and prevent early kinking of bypassed renal vessels during viscera derotation.

Anatomical contraindications for the use of the GHVG do exist: target artery larger than $9 \mathrm{~mm}$ or smaller than $5 \mathrm{~mm}$, the presence of anomalous major collaterals of the target artery originating in its proximal tract, and the presence of a severe stenosis of the distal tract of the target artery.

\subsection{Operative Technique}

With the patient positioned in a right lateral decubitus, thoraco-phreno-laparotomy is performed, and the thoracoabdominal aorta is exposed following our standard surgical technique [20]. After institution of LHBP and with a sequential clamping technique, proximal anastomosis is performed, and then critical intercostal arteries are reattached. The distal clamp is then moved below the renal arteries and the visceral aorta is opened. Using 9 Fr irrigation-occlusion catheters (LeMaitre Vascular) into the celiac trunk and the superior mesenteric artery, visceral normothermic hematic perfusion is then restored by the pump (400 mL/min). Finally, selective perfusion of the renal arteries is performed with cold $\left(4^{\circ} \mathrm{C}\right)$ crystalloid solution enriched with histidine-tryptophan-ketoglutarate (Custodiol) [31]. When a

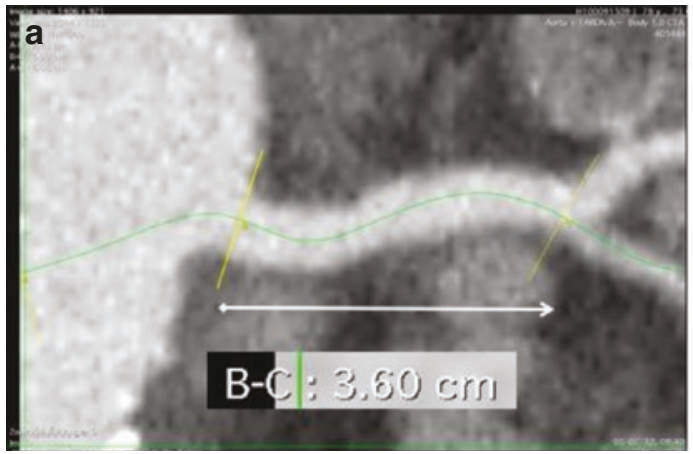

Fig. 19.2 CT scan measurement of the length of the renal artery, before the origin of a main collateral vessel. (a) Straight renal artery for $3.6 \mathrm{~cm}$, available for GHVG separate reattachment for the visceral arteries is not required, they are usually reimplanted first by means of a Carrel patch. Then, the distal aortic anastomosis is performed, and the aortic clamps are removed.

After identification of the GHVG-target vessel, the perfusion catheter is temporarily removed. A flexible steerable J tip guidewire (usually $0.035^{\prime \prime}$ ) is inserted into the renal artery, and then the constrained stented segment of the GHVG is gently placed into the artery for $2-3 \mathrm{~cm}$, with respect to the distances measured at preoperative CTA (Fig. 19.2).

Sizing of the nitinol-reinforced section was performed using a 10-20\% oversizing compared to the real diameter of the target renal vessel, as measured at preoperative CTA and confirmed by intraoperative finding. The distance between the origin and the bifurcation of the renal artery is always measured at preoperative CTA, in order to avoid unintentional coverage of renal artery branches during GHVG deployment.

The deployment line is maintained facing upward. The stent is released pulling the deployment line parallel to the vascular graft section. Great attention must be taken during these phases to hold the stented portion fixed in the correct position, avoiding to pull it deeply into the artery or retracting the graft leaving an inadequate overlapping with the artery.

Stent post-dilatation is always performed after GHVG distal segment deployment, using a 5 or $6 \mathrm{~mm}$ non-compliant balloon advanced on the guidewire through the graft. After ballooning, a

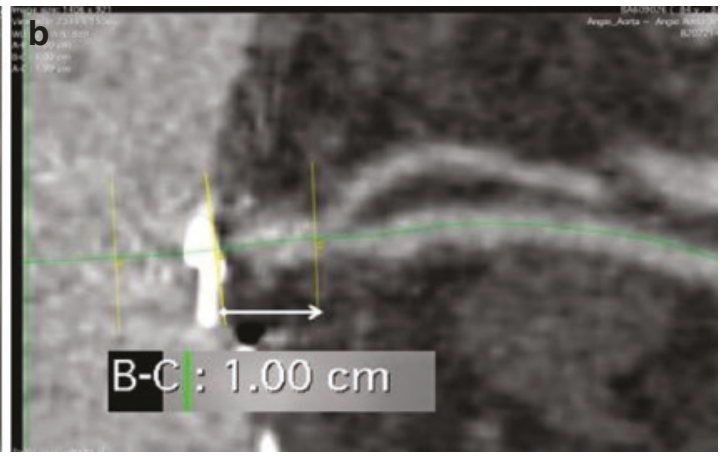

employment; (b) early collateral vessel, arising $1 \mathrm{~cm}$ from the ostium, not suitable for GHVG 

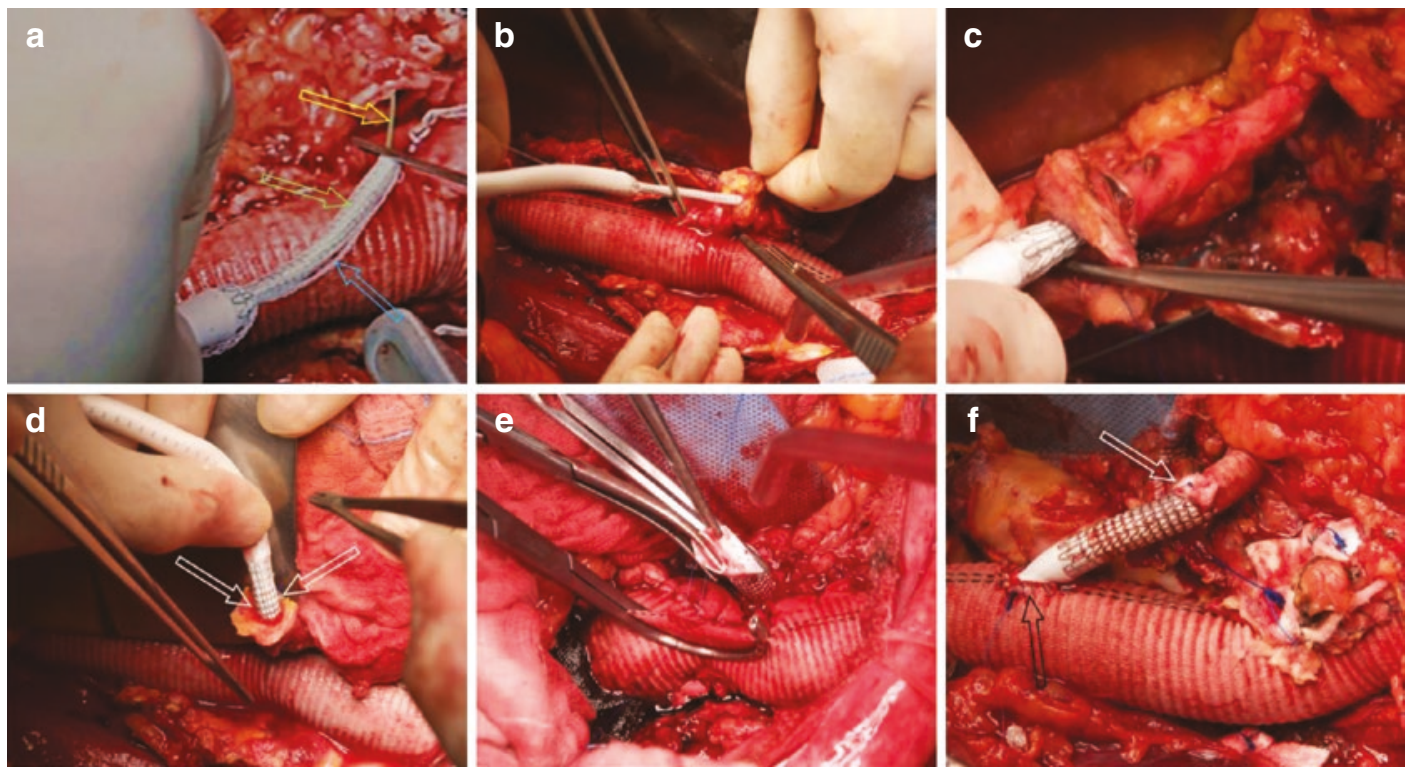

Fig. 19.3 Sequence of GHVG deployment to perform revascularization of a left renal artery. (a) Under vision, a $0.035^{\prime \prime}$ flexible steerable $\mathrm{J}$ tip guidewire (yellow arrow) facilitates the insertion of the nitinol-reinforced segment of the GHVG (green arrow) into the renal artery; (b) the nitinol-reinforced segment is gently placed into the artery for the appropriate length; (c) the stent is released pulling the deployment line (blue arrow-(a)); (d) after the

cold renal perfusion catheter is then immediately reinserted into the graft to reduce renal ischemia. To avoid accidental retraction or dislodgment of the stented portion, the GHVG is then sewn in place with at least four single circumferential monofilament polypropylene stitches. Finally, proximal anastomosis to the main aortic graft is completed in the usual fashion after cutting the proximal unstented section of the graft at the proper length. Particular care must be taken by choosing the length of the graft and the position of the proximal anastomosis to avoid kinking or compression of the unstented segment.

After blood flow restoration, protamine is administered to completely reverse heparin. Red blood cells, platelets, and plasma transfusions are aggressively used to correct severe thrombocytopenia and coagulation derangements. Anastomoses are carefully checked for bleeding and reinforced as needed. Mild initial bleeding from ePTFE needle holes usually resolved spontaneously after few minutes. In case of major release, the nitinol-reinforced segment is fixed sewing to the artery with two or four circumferential stiches (white arrows); (e) after tangential cross-clamp of the main aortic graft, the proximal anastomosis is performed; (f) final result of renal revascularization with GHVG: to notice the distal fixing stiches (white arrow) and the proximal anastomosis on the main aortic graft (black arrow)

suture-hole bleeding, hemostasis is obtained by manual compression with surgical gauze and oxidized cellulose pads (Fig. 19.3).

Antiplatelet therapy (usually aspirin) is routinely initiated by the third postoperative day, if not contraindicated. Double antiplatelet therapy (adding ticlopidine or clopidogrel) is then started by the tenth postoperative day and continued after discharge for at least 1 month.

\subsection{San Raffaele Hospital Experience}

A series of 146 consecutive patients who underwent elective TAAA open repair, including revascularization with the GHVG of at least 1 of the celiac trunk (CT), the superior mesenteric artery (SMA), and the right renal (RRA) or left renal artery (LRA) between September 2012 and September 2017, was included in this analysis. During this period, 288 other patients were submitted 
to TAAA open repair using standard renal or visceral revascularization (SRVR) techniques (such as inclusion in the Carrel patch or as a selective revascularization with a Coselli graft or an isolated standard bypass graft), because the GHVG was deemed contraindicated or not necessary by the operating surgeon (Table 19.1).

At preoperative CTA, all GHVG patients had patent CT and SMA, 140 patients had 2 functioning kidneys with both patent renal arteries, while 6 patients had only 1 functioning kidney, for a total of 578 patent visceral vessels in this group. Visceral artery stenosis was defined as a $>50 \%$ lesion as documented by CTA. Acute renal failure was defined as both a doubling of serum

Table 19.1 Other main preoperative characteristics of 146 patients submitted to TAAA open repair, including at least one vessel revascularization with the GHVG

\begin{tabular}{|c|c|}
\hline Variables & Patients $N(\%)$ \\
\hline Total & 146 \\
\hline Gender (male) & $105(72 \%)$ \\
\hline Age, $y$, mean \pm SD & $66.7 \pm 8.2$ \\
\hline \multicolumn{2}{|l|}{ Etiology } \\
\hline Atherosclerotic/degenerative & $95(65 \%)$ \\
\hline Chronic type B dissection & $45(31 \%)$ \\
\hline Para-anastomotic pseudoaneurysm & $6(4 \%)$ \\
\hline Connective tissue disorder & $0(0 \%)$ \\
\hline \multicolumn{2}{|l|}{ Classification of aneurysms ${ }^{\mathrm{a}}$} \\
\hline TAAA extent I & $10(7 \%)$ \\
\hline TAAA extent II & $61(42 \%)$ \\
\hline TAAA extent III & $32(22 \%)$ \\
\hline TAAA extent IV & $40(27 \%)$ \\
\hline TAAA extent $\mathrm{V}$ & $3(2 \%)$ \\
\hline $\begin{array}{l}\text { Aneurysm maximum diameter, } \mathrm{mm} \text {, } \\
\text { mean } \pm \mathrm{SD}\end{array}$ & $65 \pm 21$ \\
\hline $\begin{array}{l}\text { Pararenal aortic diameter, } \mathrm{mm} \text {, } \\
\text { mean } \pm \mathrm{SD}\end{array}$ & $58 \pm 18$ \\
\hline \multicolumn{2}{|l|}{ CKD stages } \\
\hline Stage I & $45(31 \%)$ \\
\hline Stage II & $63(43 \%)$ \\
\hline Stage III & $29(20 \%)$ \\
\hline Stage IV & $9(6 \%)$ \\
\hline Stage V & $0(0 \%)$ \\
\hline ASA score \pm SD & $3.5 \pm 0.5$ \\
\hline
\end{tabular}

${ }^{a}$ According to the Crawford-Safi revised TAAA extent classification

TAAA thoracoabdominal aortic aneurysm, GHVG Gore Hybrid Vascular Graft, $S D$ standard deviation, $C K D$ chronic kidney disease, ASA American Society of Anesthesiologists creatinine level and an absolute value greater than $3.0 \mathrm{mg} / \mathrm{dL}$ [32] (Fig. 19.4).

All CTAs performed after the operation were carefully reviewed in order to ascertain patency of the visceral vessels and of the implanted grafts and identify graft migration, stenosis, kinking or twisting, stent fractures, and instances of bleeding or renal/bowel infarctions.

In the analyzed period, at least one GHVG was used for revascularization in $33.6 \%$ of patients submitted to TAAA open repair. In this group of patients, among the 286 patent renal arteries, 134 were revascularized with the GHVG (96 left and 38 right), and 152 using standard surgical techniques (110 by means of Carrel patch reimplantation and 42 by means of conventional aorto-renal bypass, including Coselli graft technique). Among 140 patent CTs and 140 patent SMAs, 9 CTs and 11 SMAs were revascularized with the GHVG, respectively; the others were all

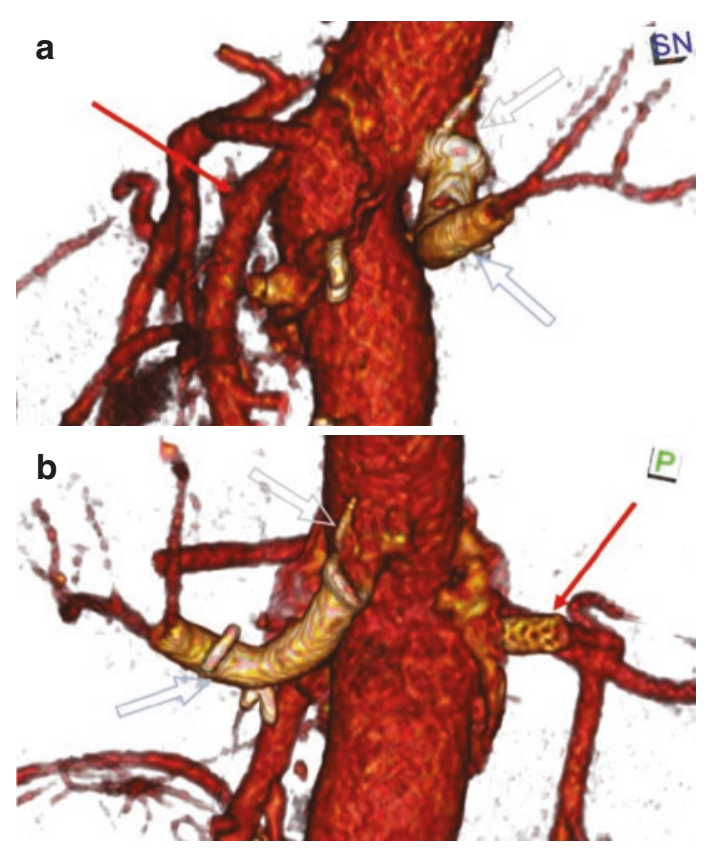

Fig. 19.4 Three-dimensional reconstruction of follow-up CT scan following TAAA open repair associated with aorto-left renal artery bypass using the GHVG. In this left view (a) and posterior view (b) of a 3D reconstruction, we should notice the ePTFE segment (gray arrow) of the GHVG, anastomosed to the main aortic graft, and the nitinol-reinforced segment (blue arrow) into the left renal artery. The small red arrow shows a bare metal stent into the right renal artery, included in Carrel patch 
included in a Carrel patch or revascularized with a Coselli graft (68\% and 32\%, respectively).

Indications to the use of the GHVG included intraoperative detection of a remote location of the ostium of the visceral artery (76\% of cases); poor-quality vessel walls, such as severe atherosclerotic degeneration or dissection $(52 \%$ of cases); and visceral artery stenosis (20\% of cases).

All intended target vessels were treated without technical concerns with the GHVG. No significant bleeding from ePTFE needle holes or from the graft anastomoses was observed. Total surgical and total renal ischemic times were $250 \pm 79 \mathrm{~min}$ and $49 \pm 23 \mathrm{~min}$, respectively. Cold renal perfusional time resulted $27 \pm 11 \mathrm{~min}$. No additional unplanned endovascular or surgical procedures were required on the GHVG-treated vessels. Prior to revascularization, a balloonexpandable stent was directly implanted at the ostium of 26 visceral arteries (6.3\% of nonGHVG revascularized visceral arteries).

In the GHVG group, 30-day mortality was $5.4 \%$ (eight patients), including one patient who died on postoperative day 6 due to myocardial infarction, two patients who died due to coagulopathy and bleeding, and five other patients who suffered from multi-organ failure. Perioperative peak decrease of the eGFR was significantly lower in the GHVG group $\left(26 \pm 18 \mathrm{~mL} / \mathrm{min} / 1.73 \mathrm{~m}^{2}\right.$ vs $\left.37 \pm 22 \mathrm{~mL} / \mathrm{min} / 1.73 \mathrm{~m}^{2}, P=0.034\right)$.

Perioperative complications included acute renal failure, occurring in 13 patients $(8 \%)$ and requiring dialysis in 5 (temporary dialysis in 4 cases and permanent dialysis in 1). Occlusion of the GHVG at subsequent CTA was found to be the main causative factor of renal failure in four of these patients. In one case, early thrombosis of a right renal GHVG was found on postoperative day 8 and then successfully treated by means of urgent endovascular recanalization with stenting PTA of the distal endpoint. No cases of GHVGrelated bowel ischemia were observed in our series (Table 19.2).

Follow-up was performed with CTA scan and clinical investigation. Patency rate at mean 22 months was 92\% (141 grafts) for the aortorenal grafts performed with the GHVG, 91\% for the contralateral renal arteries revascularized
Table 19.2 Short-term outcomes of GHVG patients

\begin{tabular}{l|l}
\hline Variables & Patients $N(\%)$ \\
\hline Total & 146 \\
\hline $\begin{array}{l}\text { Perioperative peak decrease of eGFR, } \\
\mathrm{mL} / \mathrm{min} / 1.73 \mathrm{~m}^{2}, \text { mean } \pm \mathrm{SD}\end{array}$ & $26 \pm 18$ \\
\hline Acute renal failure & $13(8 \%)$ \\
\hline Temporary hemodialysis & $4(3 \%)$ \\
\hline Dialysis at discharge & $1(1 \%)$ \\
\hline Respiratory insufficiency & $69(47 \%)$ \\
\hline Paraplegia/paraparesis & $13(8 \%)$ \\
\hline Cardiac complication & $13(8 \%)$ \\
\hline Bleeding requiring re-intervention & $3(2 \%)$ \\
\hline Operative mortality & $0(0 \%)$ \\
\hline In-hospital mortality & $7(5 \%)$ \\
\hline 30-day mortality & $7(5 \%)$ \\
\hline
\end{tabular}

GHVG Gore Hybrid Vascular Graft, $S D$ standard deviation, $e G F R$ estimated glomerular filtration rate

with standard techniques in the same group, and $92 \%$ for revascularized renal vessels in the SRR group. The observed 13 occluded GHVGs were most of all left aorto-renal bypass grafts (12 graft for LRA and 1 for RRA). A kinking/twisting of the non-stented segment was observed in eight cases, and a compressed/narrowed lumen of the stented segment was observed in five cases. No areas of renal infarction were observed in patients with patent aorto-renal grafts.

No other GHVG-related complications, reintervention, or cases of new-onset renal failure requiring dialysis were reported at follow-up in available patients.

\subsection{Gore Hybrid Vascular Graft in Hybrid Treatment}

For TAAA treatment, in addition to the standard open surgical approach and the most innovative total endovascular solution, a third option may exist: the hybrid intervention merges open surgery with endovascular options.

This procedure consists of the creation of the distal neck to allow the delivery of a thoracic endoprosthesis with the rerouting of the visceral and renal vessels through multiple selective bypasses; after an abdominal access, selective and retrograde bypasses for each abdominal vessel are created, starting from the iliac vessels or from the distal part of the abdominal aorta. 
The main advantage of this intervention is to avoid thoracotomy with a consequent reduction of respiratory complications and heart overload due to proximal aortic cross-clamping.

As written above, Lachat describes firstly his experience with VORTEC technique in the hybrid treatment of TAAAs. The natural evolution of this procedure is the use of the hybrid graft, in order to reduce the invasiveness of the procedure.

Placement of the GHVG may occur in all of the visceral and renal vessels, provided that the dimensional criteria are respected and the GHVG placement did not undergo occlusion of the early origin collateral vessels. Most of all, the renal artery and superior mesenteric artery are involved in this aspect: intentional coverage of a collateral renal artery that arises close to the aortic renal ostium may cause variable loss of renal functional parenchyma; furthermore, the middle colic artery and inferior pancreaticoduodenal artery arising in the first $2 \mathrm{~cm}$ of SMA still represent a contraindication to the use of the GHVG in order to avoid bowel hypoperfusion.

Usefulness of GHVG is represented by:

- Lack of need for extensive vessel exposure

- Faster anastomosis, even in uncomfortable anatomic situations

- Reduction of complications connected to the extensive vessel exposure (such as pancreatitis, venous or biliary tract lesions)

- Reduction of cross-clamping time and, accordingly, reduction of ischemic time

The course of GHVG into the abdomen is variable, in order to avoid kinking or compression of the graft upon viscera derotation.

Proximal anastomosis on the aortoiliac segment may be obtained with single and selective graft suture to the in-flow segment or through the creation of bifurcated/trifurcated graft with a common origin.

\subsection{Conclusions}

The specific indications to the use of GHVG included the "most feared" situations of the renal and splanchnic arteries (remote access to the ostium, poor quality of the vessel wall, ostial stenosis, or dissection), leading to the use of this device in about one third of TAAA-operated patients.

A well-known problem with ePTFE grafts, the potential prolonged needle-hole bleeding from the anastomosis, may be exacerbated by heparin, hypothermia, thrombocytopenia, and coagulopathy in TAAA open repair. Suture-line bleeding has been reported to potentially increase operative time, overall blood loss, and risk of infective complications and may require the use of a variety of topical hemostatic agents or sealants [33]. In our experience, mild initial bleeding from ePTFE needle holes resolved spontaneously after few minutes in most cases. In cases of major bleeding, we rapidly obtained hemostasis by manual compression with surgical gauze and oxidized cellulose pads. At the same time, complete reversal of heparin and aggressive transfusions are required to correct thrombocytopenia and coagulation derangements.

Our currently updated experience confirmed that technical feasibility and short-term patency rate of the GHVG for renal and visceral revascularization during TAAA open repair were satisfactory, and we hope that this novel graft will represent an additional step forward in the fight against kidney perioperative injury. Our initial results indicate that this technique is feasible also in challenging anatomies, with several potential technical advantages. Short-term clinical and radiological outcomes were satisfactory. Larger series and longer follow-up are needed to confirm the safety and durability of the proposed technique.

\section{References}

1. Lemaire SA, Jones MM, Conklin LD, Carter SA, Criddell MD, Wang XL, et al. Randomized comparison of cold blood and cold crystalloid renal perfusion for renal protection during thoracoabdominal aortic aneurysm repair. J Vasc Surg. 2009;49(1):11-9.

2. Bensley RP, Curran T, Hurks R, Lo RC, Wyers MC, Hamdan AD, et al. Open repair of intact thoracoabdominal aortic aneurysms in the American College of Surgeons National Surgical Quality Improvement Program. J Vasc Surg. 2013;58:894. https://doi. org/10.1016/j.jvs.2013.03.037. 
3. van Kuijk JP, Flu WJ, Chonchol M, Hoeks SE, Winkel TA, Verhagen HJ, et al. Temporary perioperative decline of renal function is an independent predictor for chronic kidney disease. Clin J Am Soc Nephrol. 2010;5(7):1198-204.

4. Lachat M, Mayer D, Criado FJ, Pfammatter T, Rancic Z, Genoni M, et al. New technique to facilitate renal revascularization with use of telescoping self-expanding stent grafts: VORTEC. Vascular. 2008;16(2):69-72.

5. Fehrenbacher JW, Corvera JS. Best surgical option for thoracoabdominal aneurysm repair - the open approach. Ann Cardiothorac Surg. 2012;1(3):334-8.

6. Schepens MA, Heijmen RH, Ranschaert W, Sonker U, Morshuis WJ. Thoracoabdominal aortic aneurysm repair: results of conventional open surgery. Eur J Vasc Endovasc Surg. 2009;37(6):640-5.

7. Piazza M, Ricotta JJ 2nd. Open surgical repair of thoracoabdominal aortic aneurysms. Ann Vasc Surg. 2012;26(4):600-5.

8. Panneton JM, Hollier LH. Nondissecting thoracoabdominal aortic aneurysms: part I. Ann Vasc Surg. 1995;9(5):503-14.

9. Svensson LG, Crawford ES, Hess KR, Coselli JS, Safi HJ. Experience with 1509 patients undergoing thoracoabdominal aortic operations. J Vasc Surg. 1993;17(2):357-68; discussion 368-70.

10. Back MR, Bandyk M, Bradner M, Cuthbertson D, Johnson BL, Shames ML, et al. Critical analysis of outcome determinants affecting repair of intact aneurysms involving the visceral aorta. Ann Vasc Surg. 2005;19(5):648-56.

11. Dubois L, Durant C, Harrington DM, Forbes TL, Derose G, Harris JR. Technical factors are strongest predictors of postoperative renal dysfunction after open transperitoneal juxtarenal abdominal aortic aneurysm repair. J Vasc Surg. 2013;57(3):648-54.

12. Crawford ES, Snyder DM, Cho GC, Roehm JO Jr. Progress in treatment of thoracoabdominal and abdominal aortic aneurysms involving celiac, superior mesenteric, and renal arteries. Ann Surg. 1978;188(3):404-22.

13. Tshomba Y, Melissano G, Civilini E, Setacci F, Chiesa R. Fate of the visceral aortic patch after thoracoabdominal aortic repair. Eur J Vasc Endovasc Surg. 2005;29(4):383-9.

14. Tshomba Y, Bertoglio L, Marone EM, Melissano G, Chiesa R. Visceral aortic patch aneurysm after thoracoabdominal aortic repair: conventional vs hybrid treatment. J Vasc Surg. 2008;48(5):1083-91.

15. Kulik A, Castner CF, Kouchoukos NT. Patency and durability of presewn multiple branched graft for thoracoabdominal aortic aneurysm repair. J Vasc Surg. 2010;51(6):1367-72.

16. Black JH 3rd. Technique for repair of suprarenal and thoracoabdominal aortic aneurysms. J Vasc Surg. 2009;50(4):936-41.

17. Clouse WD, Marone LK, Davison JK, Dorer DJ, Brewster DC, LaMuraglia GM, et al. Late aortic and graft-related events after thoracoabdominal aneurysm repair. J Vasc Surg. 2003;37(2):254-61.

18. Svensson LG, Crawford ES, Hess KR, Coselli JS, Safi HJ. Thoracoabdominal aortic aneurysms associated with celiac, superior mesenteric, and renal artery occlusive disease: methods and analysis of results in 271 patients. J Vasc Surg. 1992;16:378-90.

19. Clair DG, Belkin M, Whittemore AD, Mannick JA, Donaldson MC. Safety and efficacy of transaortic renal endarterectomy as an adjunct to aortic surgery. J Vasc Surg. 1995;21:926-34.

20. Chiesa R, Melissano G, Civilini E, Bertoglio L, Rinaldi E, Marone EM, et al. Video-atlas of open thoracoabdominal aortic aneurysm repair. Ann Cardiothorac Surg. 2012;1(3):398-403.

21. LeMaire SA, Jamison AL, Carter SA, Wen S, Alankar S, Coselli JS. Deployment of balloon expandable stents during open repair of thoracoabdominal aortic aneurysms: a new strategy for managing renal and mesenteric artery lesions. Eur J Cardiothorac Surg. 2004;26(3):599-607.

22. Patel R, Conrad MF, Paruchuri V, Kwolek CJ, Cambria RP. Balloon expandable stents facilitate right renal artery reconstruction during complex open aortic aneurysm repair. J Vasc Surg. 2010;51(2): 310-5.

23. Donas KP, Lachat M, Rancic Z, Oberkofler C, Pfammatter T, Guber I, et al. Early and midterm outcome of a novel technique to simplify the hybrid procedures in the treatment of thoracoabdominal and pararenal aortic aneurysms. J Vasc Surg. 2009;50(6):1280-4

24. Donas KP, Rancic Z, Lachat M, Pfammatter T, Frauenfelder T, Veith FJ, et al. Novel sutureless telescoping anastomosis revascularization technique of supra-aortic vessels to simplify combined open endovascular procedures in the treatment of aortic arch pathologies. J Vasc Surg. 2010;51(4):836-41.

25. Bonvini S, Ricotta JJ, Piazza M, Ferretto L, Grego F. ViPS technique as a novel concept for a sutureless vascular anastomosis. J Vasc Surg. 2011;54(3): 889-92.

26. Greenberg G, Szendro G, Mayzler O, Ginzburg V, Leytzin A. Use of ViaBahn open revascularisation technique for above-knee femoro-popliteal anastomosis: a technical note. Eur J Vasc Endovasc Surg. 2011;42(2):202-5.

27. Levack MM, Bavaria JE, Gorman RC, Gorman JH 3rd, Ryan LP. Rapid aortic arch debranching using the Gore hybrid vascular graft. Ann Thorac Surg. 2013;95(6):e163-5.

28. Nigro G, Gatta E, Pagliariccio G, Grilli C, Carbonari L. Use of the Gore Hybrid Vascular Graft in a challenging high-lying extracranial carotid artery aneurysm. J Vasc Surg. 2014. https://doi.org/10.1016/j. jvs.2013.04.044.

29. Bornak A, Goldstein LJ, Rey J, Medina A, Yang JK, Velazquez OC, et al. Aortic aneurysmal repair with surtureless visceral revascularization using novel 
hybrid vascular graft and a gradual funneling technique. Vasc Endovasc Surg. 2012;46(3):258-61.

30. W. L. Gores \& Associates. Instructions for use for Gore Hybrid Vascular Graft. http://www.goremedical.com/resources/dam/assets/AP3350ML3.HYVG. US_IFU.pdf.

31. Tshomba Y, Kahlberg A, Melissano G, Coppi G, Marone E, Ferrari D, et al. Comparison of renal perfusion solutions during thoracoabdominal aortic aneurysm repair. J Vasc Surg. 2014;59:623-33.
32. Kashyap VS, Cambria RP, Davison JK, L'Italien GJ. Renal failure after thoracoabdominal aortic surgery. J Vasc Surg. 1997;26(6):949-55; discussion 955-7.

33. Saha SP, Muluk S, Schenk W 3rd, Dennis JW, Ploder B, Grigorian A, Presch I, Goppelt A. A prospective randomized study comparing fibrin sealant to manual compression for the treatment of anastomotic suturehole bleeding in expanded polytetrafluoroethylene grafts. J Vasc Surg. 2012;56(1):134-41. 